\title{
Irradiated accretion discs in post novae
}

\author{
M. R. Schreiber ${ }^{1,2}$ and B. T. Gänsicke ${ }^{1}$ \\ 1 Universitäts-Sternwarte, Geismarlandstr. 11, 37083 Göttingen, Germany \\ 2 Department of Astronomy, University of Cape Town, Private Bag, Rondebosch 7700, Cape Town, South Africa
}

Received 22 February 2001 / Accepted 13 June 2001

\begin{abstract}
We derive approximations for the $\dot{M}-\Sigma$ relation (the so-called S-curves) of irradiated accretion discs from detailed vertical structure calculations. Using these approximations, we simulate the time-dependent behaviour of irradiated accretion discs in dwarf novae and post novae. Our calculations show, in agreement with Hameury et al. (1999), that irradiation of the accretion disc should cause small "echo" outbursts immediately following normal outbursts - which contrasts with the observed dwarf nova light curves. In addition, we calculate light curves of post nova systems where the accretion disc is strongly irradiated by the hot white dwarf. We find that the visual brightness of post novae gradually decreases after the nova explosion as a result of the decreasing disc irradiation from the continuously cooling white dwarf. The rate of decline that we derive is in good agreement with the observations. While we cannot exclude that additional effects may contribute to the observed fading of post novae, e.g., a slow decrease of the accretion rate, our calculations show that irradiation from the cooling white dwarf plays an important rôle in the post nova evolution.
\end{abstract}

Key words. accretion, accretion discs - stars: binaries: close - stars: individual: V446 Her - novae, cataclysmic variables

\section{Introduction}

In non-magnetic cataclysmic variables (CVs) (see Warner 1995, for an encyclopaedic review) a white dwarf accretes hydrogen-rich material from the Roche-lobe filling secondary via an accretion disc. The sudden brightenings - called dwarf nova outbursts - observed in many CVs, are caused by thermal instabilities in the accretion disc (Cannizzo 1993b; Ludwig et al. 1994, for parameter studies).

A significant fraction of CVs, especially those with long orbital periods, contain a moderately hot $\left(T_{\mathrm{wd}} \sim\right.$ $30000-50000 \mathrm{~K}$ ) white dwarf (Gänsicke 2000; Sion 1999). Even though it is clear that irradiation from the hot white dwarf will affect the structure of the accretion disc in these systems this effect was ignored until recently by the various groups working on disc instability models. A selfconsistent description of dwarf nova outbursts of irradiated accretion discs was first presented by Hameury et al. (1999), roughly 25 years after the thermal limit cycle model was developed. The main effect on the computed light curves which results from the inclusion of irradiation is the appearence of "echo" outbursts caused by the reflection of the cooling front when it reaches the strongly irradiated inner parts of the accretion disc. This

Send offprint requests to: M. R. Schreiber,

e-mail: mschrei@uni-sw.gwdg.de result is in contrast with the long term visual light curves of well-observed dwarf nova, such as SS Cyg or U Gem, which provide no evidence for the predicted echo outbursts. Hameury et al. (1999) concluded, hence, that either the inner disc is evaporated, the efficiency of the irradiating flux from the white dwarf is essentially lower than expected previously (e.g. in King 1997), or both.

Post nova systems are excellent laboratories to study irradiated accretion discs, as the white dwarf is heated up to a peak temperature of $\gtrsim 3 \times 10^{5} \mathrm{~K}$ during a nova eruption and provides thereafter a time-dependent source of strong irradiation as it slowly cools down to its equilibrium temperature. In an earlier paper, we already demonstrated that irradiation from the hot white dwarf prevents the occurrence of dwarf nova outbursts in post nova accretion discs for up to $\sim 100$ yr (Schreiber et al. 2000a, hereafter Paper I). Here we present detailed calculations of irradiated discs in CVs and show that the decreasing irradiation from the white dwarf necessarily implies a significant decline of the visual brightness in post novae. Such a decline has indeed been observed in a large number of post novae, and has so far been widely interpreted in the context of the hibernation scenario (Shara et al. 1986) as a decrease of the mass transfer rate from the secondary star (e.g. Duerbeck 1992).

In Sect. 2 we calculate the vertical structure of irradiated accretion discs and derive useful approximations for 
the vertical equilibrium $\dot{M}-\Sigma$ curves in order to calculate the temporal evolution of irradiated accretion discs. Having compared our results with those of Hameury et al. (1999) we compute detailed long term post novae light curves, taking into account the time dependent irradiation from the cooling white dwarf (Sect. 3). Finally we derive a useful analytical expression describing the visual decline in post novae and we discuss our results in the light of the post nova evolution.

\section{Dwarf nova outbursts of irradiated accretion disc}

We calculated a grid of vertical accretion disc structures using the code written by J. K. Cannizzo and described in Cannizzo \& Wheeler (1984) and Cannizzo \& Cameron (1988), which we have modified to include the effects of external irradiation by equating the sum of the irradiation flux $\sigma T_{\text {irr }}^{4}$ and the viscous heating $F_{\text {vis }}=\sigma T_{\text {vis }}^{4}$ to the total flux emitted by each disc annulus at the outer boundary: $\sigma T_{\text {eff }}^{4}=F_{\text {vis }}+\sigma T_{\text {irr }}^{4}$ (Paper I; Hameury et al. 1999; Tuchman et al. 1990; Mineshige et al. 1990).

Using the resulting database we derive expressions for the critical accretion rates and the critical surface densities. Throughout this paper the index "+" refers to critical values of the upper, hot branch of the S-curve (below which the hydrogen in the disc starts to recombine and the disc becomes unstable) whereas the index "-_" denotes critical values of the lower, cold branch (above which the hydrogen becomes partially ionised). For the non-irradiated disc (i.e. $T_{\text {irr }}=0$ ), we obtain

$$
\begin{aligned}
& \dot{M}_{+}=9.5 \times 10^{15} \mathrm{~g} \mathrm{~s}^{-1} R_{10}^{2.64} \alpha_{\mathrm{h}}^{0.01} M_{\mathrm{wd}}^{-0.88}, \\
& \dot{M}_{-}=4.0 \times 10^{15} \mathrm{~g} \mathrm{~s}^{-1} R_{10}^{2.66} \alpha_{\mathrm{c}}^{-0.001} M_{\mathrm{wd}}^{-0.88},
\end{aligned}
$$

and

$$
\Sigma_{+}=8.0 \mathrm{~g} \mathrm{~cm}^{-2} R_{10}^{1.11} \alpha_{\mathrm{h}}^{-0.75} M_{\mathrm{wd}}^{-0.38},
$$$$
\Sigma_{-}=12.8 \mathrm{~g} \mathrm{~cm}^{-2} R_{10}^{1.14} \alpha_{\mathrm{c}}^{-0.78} M_{\mathrm{wd}}^{-0.38} \text {, }
$$

where $R_{10}=R / 10^{10}[\mathrm{~cm}], M_{\mathrm{wd}}$ the white dwarf mass in solar units and $\alpha_{\mathrm{c}}$ and $\alpha_{\mathrm{h}}$ the viscosity parameter for the cold and hot branch, respectively. Equations (1)-(4) are in agreement with earlier derived expressions (e.g. Hameury et al. 1999; Cannizzo 1993a; Ludwig et al. 1994).

The irradiation temperature $T_{\mathrm{irr}, \mathrm{s}}$ necessary to supress the instability increases with decreasing $\alpha$ and decreasing radius:

$T_{\mathrm{irr}, \mathrm{s}}=7382 \mathrm{~K} \alpha^{-0.07} R_{10}^{-0.03}$,

(Paper I). The decrease of the critical accretion rates and surface densities between the non-irradiated case and the suppression of the instability is approximately given by:

$$
\begin{aligned}
& \dot{M}_{+}^{\mathrm{irr}}=\dot{M}_{\mathrm{cr}}\left(1-\frac{T_{\mathrm{irr}}^{7.2}}{T_{\mathrm{irr}, \mathrm{s}}^{7.2}}\right), \\
& \dot{M}_{-}^{\mathrm{irr}}=\dot{M}_{\mathrm{cr}}\left(1-\frac{T_{\mathrm{irr}}^{4.5}}{T_{\mathrm{irr}, \mathrm{s}}^{4.5}}\right),
\end{aligned}
$$

and

$$
\begin{aligned}
& \Sigma_{+}^{\mathrm{irr}}=\Sigma_{+}\left(1-\frac{T_{\mathrm{irr}}^{3.7}}{T_{\mathrm{irr}, \mathrm{s}}^{3.7}}\right) \\
& \Sigma_{-}^{\mathrm{irr}}=\Sigma_{-}\left(1-\frac{T_{\mathrm{irr}}^{6.3}}{T_{\mathrm{irr}, \mathrm{s}}^{6.3}}\right)
\end{aligned}
$$

For the central temperature we find in the absence of irradiation:

$$
\begin{aligned}
& T_{\mathrm{c},+}=35500 \mathrm{~K}\left(\frac{\alpha_{\mathrm{h}}}{0.1}\right)^{-0.2} M_{\mathrm{wd}}^{-0.014} R_{10}^{0.043} \\
& T_{\mathrm{c},-}=12620 \mathrm{~K}\left(\frac{\alpha_{c}}{0.05}\right)^{-0.2} M_{\mathrm{wd}}^{-0.01} R_{10}^{0.03}
\end{aligned}
$$

which is in good agreement with Ludwig \& Meyer (1998). We find $T_{\mathrm{c},-}$ somewhat increasing but - especially in the case of low $\alpha$ - nearly independent of irradiation whereas $T_{\mathrm{c},+}$ decreases with increasing irradiation. In the calculations presented in this paper we use the following approximations:

$$
\begin{aligned}
& T_{\mathrm{c},+}^{\mathrm{irr}}=T_{\mathrm{c},+}\left(1-\frac{T_{\mathrm{irr}}^{2.5}}{T_{\mathrm{irr}, \mathrm{s}}^{2.5}}\right) \\
& T_{\mathrm{c},-}^{\mathrm{irr}}=T_{\mathrm{c},-}+\left(15850 \mathrm{~K}-T_{\mathrm{c},-}\right) \frac{T_{\mathrm{irr}}^{2.1}}{T_{\mathrm{irr}, \mathrm{s}}^{2.1}} .
\end{aligned}
$$

For the viscous heating of the photosphere on the hot branch $\left(T_{\text {vis,cold }}\right)$ we find an expression similar to that of Cannizzo (1993a) and Ludwig \& Meyer (1998) also being valid in the irradiated case:

$T_{\text {vis }, \text { hot }}=37346 \mathrm{~K}_{\mathrm{wd}}^{-1 / 8} R_{10}^{3 / 8} T_{\mathrm{c}, 5}^{2} \Sigma_{2}^{-1 / 2}$.

Here $\Sigma_{2} \equiv \Sigma / 100\left[\mathrm{~g} / \mathrm{cm}^{2}\right]$ and $T_{\mathrm{c}, 5} \equiv T_{\mathrm{c}} / 10^{5}[\mathrm{~K}]$. For the viscous heating of the photosphere at the cold optically thick branch $\left(T_{\text {vis,cold }}\right)$ we obtain

$T_{\text {vis }, \text { cold }}=5500 \mathrm{~K}\left(\frac{\Sigma}{\Sigma_{-}}\right)^{0.17}\left(\frac{T_{\mathrm{c}}}{T_{\mathrm{c},-}}\right)^{0.41} R_{10}^{-0.05}$,

where $\Sigma_{-}$is given by Eq. (4) and $T_{\mathrm{c},-}$ by Eq. (11) respectively.

For the unstable region between the critical values we use

$\log \left(T_{\mathrm{vis}}\right)=\frac{\log \left(T_{\mathrm{c}} / T_{\mathrm{c},+}\right)}{\log \left(T_{\mathrm{c},-} / T_{\mathrm{c},+}\right)} \log \left(\frac{T_{\mathrm{vis},+}}{T_{\mathrm{vis},-}}\right)+\log \left(T_{\mathrm{vis},+}\right)$

and a sharp transistion between $\alpha_{\mathrm{c}}$ and $\alpha_{\mathrm{h}}$ :

$$
\begin{aligned}
\log (\alpha)=\log \left(\alpha_{\mathrm{c}}\right) & +\left[\log \left(\alpha_{\mathrm{h}}\right)-\log \left(\alpha_{\mathrm{c}}\right)\right] \\
& \times\left[1+\left(\frac{T_{\mathrm{c}}}{0.5\left(T_{\mathrm{c},+}+T_{\mathrm{c},-}\right)}\right)^{8}\right]^{-1}
\end{aligned}
$$

similar to that used by Hameury et al. (1999).

Using Eqs. (1)-(17) ${ }^{1}$ we can simulate the behaviour of geometrically thin accretion discs in the vertical averaged

1 These approximations are somewhat rough and occasionally they can be off by $\sim 30 \%$ but, as pointed out by Hameury et al. (1998) and Dubus et al. (1999), even in a non-irradiated disc cooling fluxes are not known more accurately. 


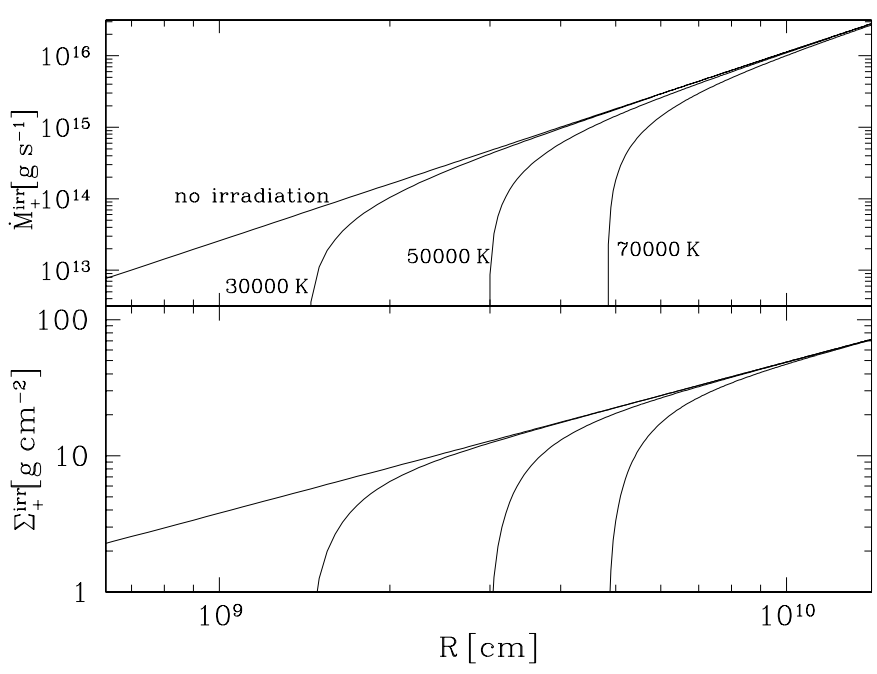

Fig. 1. The critical accretion rate $\dot{M}_{+}^{\text {irr }}$ and surface density $\Sigma_{+}^{\text {irr }}$ (lower panel) in the inner disc regions for different temperatures of the white dwarf $\left((1-\beta)^{0.25} T_{\mathrm{wd}}\right)$. The binary parameters correspond to estimates for the post nova V446 Her, i.e. $R_{\text {out }}=3.8 \times 10^{10} \mathrm{~cm}$ (the outer edge of the disc), $R_{\text {in }}=$ $6 \times 10^{8} \mathrm{~cm}$ (the inner edge of the disc), $M_{\mathrm{wd}}=0.8 M_{\odot}$, and $\alpha_{\mathrm{h}}=0.1$.

description, selfconsistently including external irradiation. In order to solve the equations of the time dependent radial evolution of the disc we take the FE-code used for the first time in Schreiber \& Hessman (1998) and described in detail in Schreiber et al. (2000b).

As in Paper I we use the description of disc irradiation given by Adams et al. (1988) and King (1997). In the following calculations we assume system parameters which are appropriate for the post nova V446 Her (Nova Herculis 1960), the best documented post nova that shows regular dwarf nova outbursts and that has a typical CV orbital period of $5 \mathrm{hr}$. Specifically, we use $R_{\text {out }}=3.8 \times 10^{10} \mathrm{~cm}$, $M_{\mathrm{wd}}=0.8 M_{\odot}, R_{\mathrm{in}}=6 \times 10^{8} \mathrm{~cm}, \alpha_{\mathrm{h}}=0.1, \alpha_{\mathrm{c}}=0.02$, $\log \left(M_{\mathrm{tr}}\left[\mathrm{g} \mathrm{s}^{-1}\right]\right)=16.8, d=1 \mathrm{kpc}$, and an inclination of $i=50^{\circ}$.

Figure 1 shows the effect of irradiation on the critical accretion rate and surface density $\left(\dot{M}_{+}^{\text {irr }}, \Sigma_{+}^{\text {irr }}\right)$ for different temperatures of the white dwarf. We did not include the boundary layer luminosity because we were mainly interested in post nova light curves in which irradiation of the extremely hot white dwarf dominates the irradiating flux. The influence of both the white dwarf and the boundary layer luminosity, in the case of moderately hot white dwarfs ( $20000 \mathrm{~K})$, has been discussed by Hameury et al. (1999, 2000). Figure 2 shows light curves for different amounts of disc irradiation. To determine the visual brightness $V$, we use the prescription of Cannizzo (1993a) but scaled for the larger distance and used $T_{\mathrm{eff}}^{4}=T_{\mathrm{vis}}^{4}+T_{\mathrm{irr}}^{4}$ instead of $T_{\text {eff }}^{4}=T_{\text {vis }}^{4}$.

Our results confirm the finding of Hameury et al. (1999) that irradiation causes small post-eruption outbursts. These outbursts occur because the cooling wave gets reflected when reaching the inner parts of the disc where the instability is suppressed.

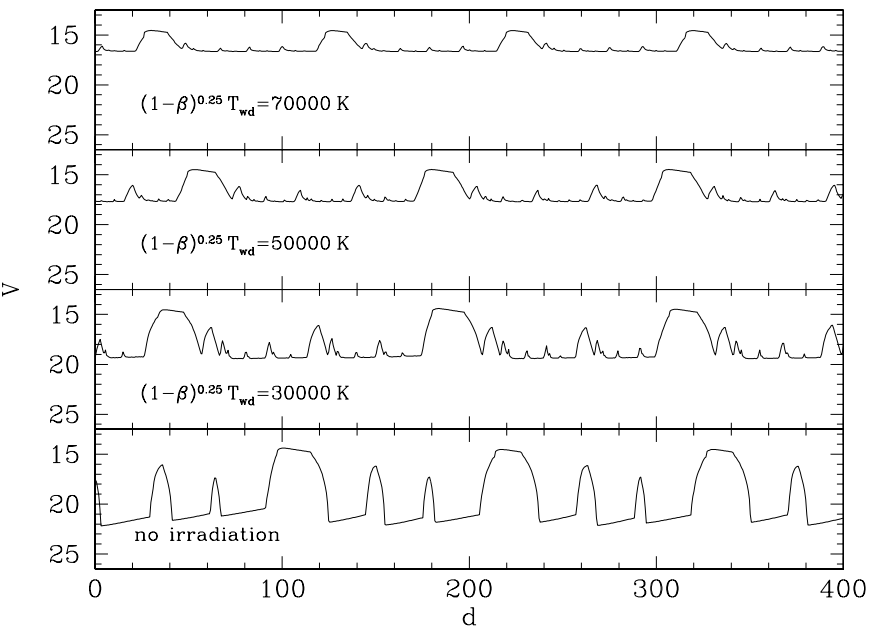

Fig. 2. Light curves for different amounts of irradiation using system parameters appropriate for V446 Her. Because of the irradiation, there is always an unstable region in the disc causing small "echo" outbursts immediately following normal outbursts. In the case of very strong irradiation (upper panel) these small outbursts are hardly seen because the visual brightness of the irradiated disc outshines most of these small outbursts.

This clearly shows that there exists an unstable transition region in the disc also during quiescence resulting in heating and cooling fronts traveling through the disc and producing small outbursts. In the light curves of Fig. 2 most of these small outbursts cannot be discerned because they affect only a small area of the disc and do not contribute much to the overall brightness of the strongly irradiated disc.

Our light curve obtained for $(1-\beta) T_{\mathrm{wd}}=30000$ (third panel from top in Fig. 2) is very similar to the light curve presented by Hameury et al. (1999) (upper panel of their Fig. 3) using $M_{\mathrm{wd}}=0.6 M_{\odot}$ and low mass transfer rates. Notice that although our numerical calculations do not consider radius variations they are qualitatively correct because irradiation of the disc makes it difficult to obtain outside-in outbursts (Hameury et al. 1999).

\section{Post novae light curves}

In Paper I we compared our estimates of irradiation suppression of dwarf nova outbursts with the photometric history of V446 Her. We found the picture of time dependent irradiation due to the cooling white dwarf to be in agreement with the (relatively sparse) observations of V446 Her. Our calculations showed also that irradiation should still keep the innermost parts of the disc in the hot ionised state today.

Here, we simulate in detail the dwarf nova outbursts in post novae using the method described in the previous section and a simple power-law for the cooling of the white dwarf

$L_{\mathrm{wd}} \propto t^{-1}$, 

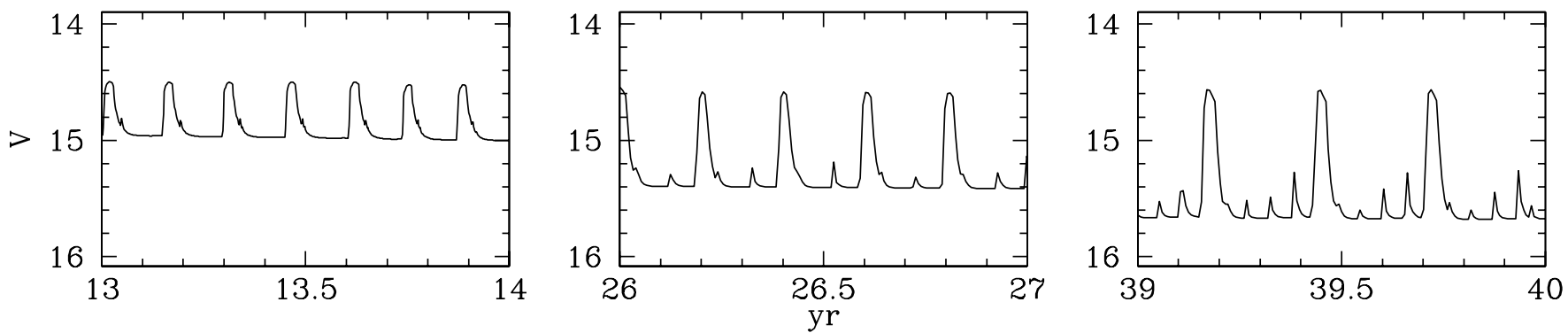

Fig. 3. Snapshots of dwarf nova outbursts from our long-term simulation shown in Fig. 4a. The left panel corresponds to $T_{\mathrm{wd}} \simeq 1.6 \times 10^{5} \mathrm{~K}$ and the outburst magnitude is only $0.5 \mathrm{mag}$. The cooling wave gets repeatedly reflected between the hot inner part of the disc and the disc edge, resulting in an exponential decline superimposed by small dips. After 26 years the white dwarf has cooled down to $T_{\mathrm{wd}} \simeq 1.3 \times 10^{5} \mathrm{~K}$ and small echo outbursts appear between the larger ones. Today ( $\sim 40 \mathrm{yr}$ after turn-off), $T_{\mathrm{wd}} \simeq 1.2 \times 10^{5} \mathrm{~K}$ and the small outbursts caused by multiple reflections of the heating and cooling waves become increasingly pronounced.

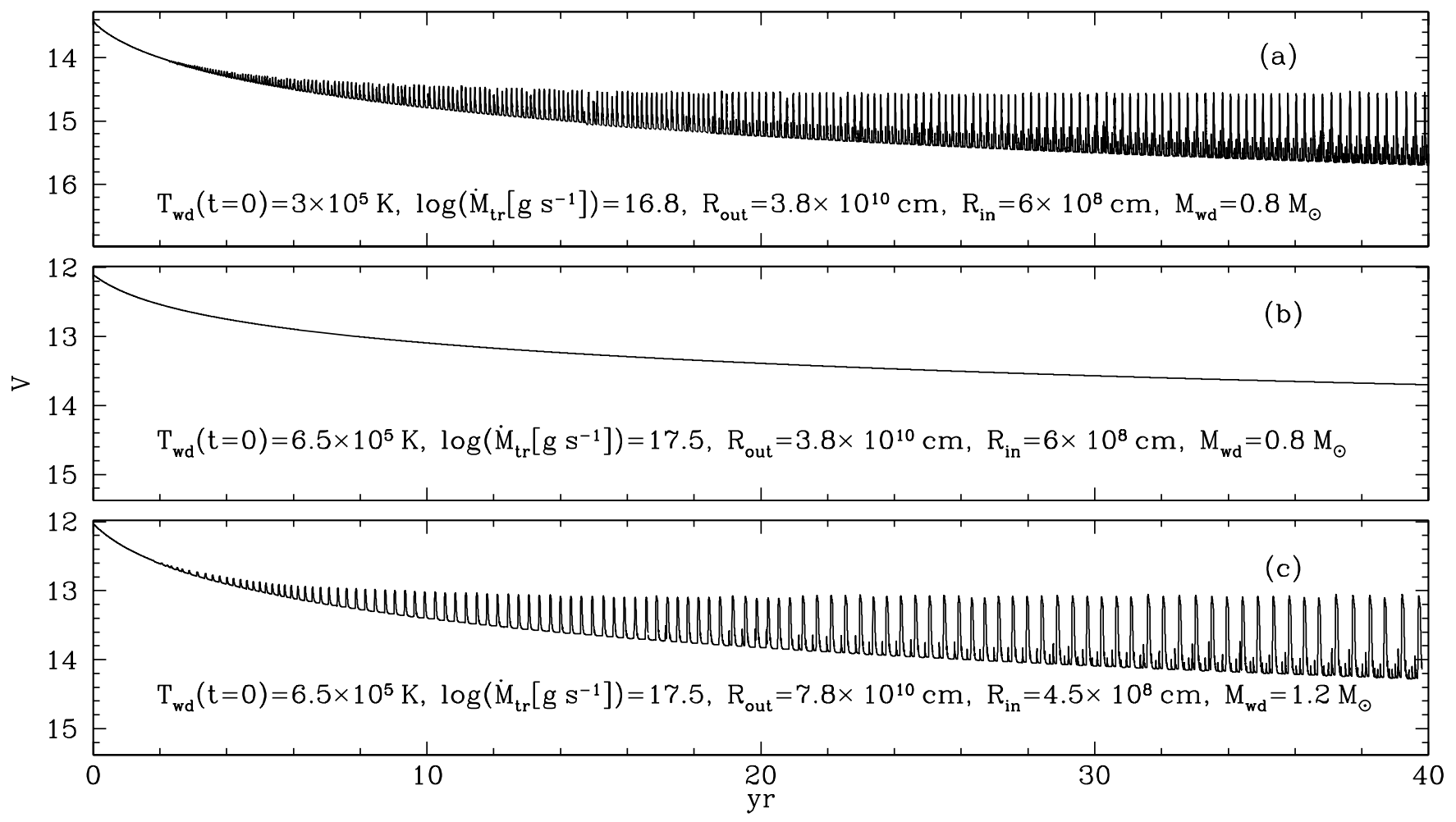

Fig. 4. Simulations of the occurrence of dwarf nova outbursts in post novae assuming moderate and constant mass transfer, a disc albedo of $\beta=0.5, \alpha_{\mathrm{h}}=0.1, \alpha_{\mathrm{c}}=0.02$ and $L_{\mathrm{wd}} \propto t^{-1}$ as cooling law for the white dwarf (see Eq. (20)). Panel a) displays the light curve most realistic for V446 Her: the outbursts start $~ 5$ yr after the hydrogen burning turned off and the magnitude of the outbursts today $(1960+40)$ is around 1.5 mag. Panel b) displays the extremest case of irradiation that we considered: we assume the same geometry as in a), but a turn-off temperature $T_{\mathrm{wd}}(t=0)=6.5 \times 10^{5} \mathrm{~K}$. As a result, the thermal instability is suppressed for many decades. The influence of the disc radius (i.e., the orbital period) is shown in panel c): even in the case of an extremely heated white dwarf and a high accretion rate, such a large disc disc produces outbursts already $\sim 5$ yr after the hydrogen burning turned off.

(Prialnik 1986; Somers \& Naylor 1999). Using the approximations derived in Sect. 2 to calculate the time dependent evolution of irradiated discs in post nova, and the time dependent irradiation resulting from Eq. (18), and assuming a disc albedo of $\beta=0.5$, we considered the following three cases:

(a) We used the system parameters of V446 Her (Sect. 2) and a turn-off temperature of $3 \times 10^{5} \mathrm{~K}$. Even for such a conservatively low turn-off temperature, the white dwarf in V446 Her is today - 40 years after the nova explosion - still very hot, $\sim 1.2 \times 10^{5} \mathrm{~K}$. The simulated light curve displayed in Figs. 3 and 4 a qualitatively ${ }^{2}$ reproduces the observations of Stienon (1971) and Honeycutt et al. (1998): between September 1968 and September 1970 the system was observed consistently at $m_{\mathrm{pg}} \sim 15.8$ and in

2 Note that we did not apply any interstellar extinction. Thorstensen \& Taylor (2000) estimate $A_{V} \sim 1$ mag. 
1998 regular dwarf nova outbursts of $2.5 \mathrm{mag}$ ( $V$ between 18 and 15.5) were observed (Honeycutt et al. 1998).

Considering the relatively poor sampling of the observed light curve of V446 Her and the uncertainties in the turn-off time, in the system parameters $\left(M_{\mathrm{wd}}, M_{\mathrm{sec}}, \dot{M}_{\mathrm{tr}}\right.$, $d, i)$, and, most importantly, in the disc albedo $\beta$, we did not attempt to fit quantitatively the current magnitude and outburst frequency of V446 Her. However, once a detailed observed light curves of V446 Her resolving the actual shape of the dwarf nova outbursts will be available, such a quantitative comparison will be a crucial test for the theory of the thermal instability in irradiated accretion discs and a promising way to estimate the efficiency of disc irradiation, i.e. $\beta$. Note that even if the reprocessing efficiency is as low as, e.g., the calculations by Suleimanov et al. (1999) suggest (they find $\beta \sim 0.93$ for irradiated accretion discs in supersoft X-ray sources), irradiation by the hot white dwarf in V446 Her should still noticeably influence the outburst behaviour in this system. For $\beta=0.93$ and a white dwarf temperature of $T_{\mathrm{wd}}=1.2 \times 10^{5} \mathrm{~K}$, the corresponding "effective irradiation" temperature is

$(1-\beta)^{0.25} T_{\mathrm{wd}} \simeq 50000 \mathrm{~K}$

and the resulting light curve contains detectable echo outbursts (Fig. 2, second panel from top).

(b) We used again the system parameters of V446 Her, but a much higher turn-off temperature of $T_{\mathrm{wd}}(t=0)=$ $6.5 \times 10^{5} \mathrm{~K}$ and significantly higher mass transfer, i.e. $\log \left(M_{\mathrm{tr}}\left[\mathrm{g} \mathrm{s}^{-1}\right]\right)=17.5$ instead of $\log \left(M_{\mathrm{tr}}\left[\mathrm{g} \mathrm{s}^{-1}\right]\right)=16.8$, to calculate the visual decline and the supression of disc outbursts under extreme conditions (Fig. 4b). In this case the supression of disc instabilities due to irradiation lasts many decades.

(c) Our last simulation shows the light curve produced by a larger accretion disc $\left(R_{\text {out }}=7.8 \times 10^{10} \mathrm{~cm}\right)$ around a more massive but again very hot primary $\left(M_{\mathrm{wd}}=1.2 M_{\odot}\right.$, $\left.T_{\mathrm{wd}}(t=0)=6.5 \times 10^{5} \mathrm{~K}\right)$, corresponding to a system with a long orbital period, $P \sim 15 \mathrm{hr}$ (Fig. 4c). The occurrence of dwarf nova outbursts is prevented for only $\sim 5 \mathrm{yr}$. The duration of the outbursts and the quiescence intervals are, as expected, longer than for smaller discs. Hence, for a long-period post nova such as GK Per (Nova Per 1901), as a result of the larger disc size, even for a very high turnoff temperature dwarf nova outbursts are hardly supressed at all.

Our long term simulation of post novae (Fig. 4) shows that the decreasing disc irradiation from the cooling white dwarf results in a slow decrease of the optical brightness. The computed visual decline can be approximated with a simple power-law, i.e. $\mathrm{d} M_{V} / \mathrm{d} \log t \simeq 1$. This result is easy to understand by considering the following simple but analytical estimate of the visual decline resulting from the decreasing irradiation. Smak (1989) calculated the absolute magnitudes $M_{V}$ of an accretion disc as a function of the accretion rate, the mass of the central star, and the radius of the disc. At high accretion rates, $\dot{M}_{\text {acc }} \gtrsim 10^{17} \mathrm{~g} \mathrm{~s}^{-1}$, he finds:

$$
\frac{\mathrm{d} M_{V}}{\mathrm{~d} \log \dot{M}_{\mathrm{acc}}} \simeq-1 \text {. }
$$

For the radial dependence of disc irradiation by the white dwarf King (1997) derived for $R \gg R_{\mathrm{wd}}$ :

$T_{\text {irr }} \propto R^{-3 / 4}$.

The viscous heating of the disc's photosphere $T_{\text {vis }}$ in a steady thin accretion disc has the same radial dependence:

$T_{\text {vis }} \propto R^{-3 / 4} \propto \dot{M}_{\text {acc }}^{1 / 4}$.

Therefore we can estimate the decline of the visual brightness due to the decreasing effect of disc irradiation by the (cooling) hot white dwarf replacing the accretion rate with the luminosity of the white dwarf (note: $T_{\mathrm{vis}}^{4} \propto \dot{M}, T_{\mathrm{irr}}^{4} \propto$ $\left.L_{\mathrm{wd}}\right)$ in Smak's relation, Eq. (22). The time dependence of the white dwarf luminosity is given by Eq. (20)

$\frac{\mathrm{d} \log L_{\mathrm{wd}}}{\mathrm{d} \log t}=-1$

and we obtain:

$\frac{\mathrm{d} M_{V}}{\mathrm{~d} \log t} \simeq 1$

This simple estimate is in good agreement with the visual decline computed from our detailed light curve simulations (Fig. 5).

It appears, therefore, unavoidable that the decreasing irradiation from the slowly cooling white dwarf in post novae will result in an observable decline of the visual brightness over many decades after the nova explosion. Indeed, the data compiled by Duerbeck (1992) for 13 post novae, covering observations obtained 24 to 101 years after their nova explosions, give a mean decline rate of $\mathrm{d} M_{V} / \mathrm{d} t=0.01{\text { mag } \mathrm{yr}^{-1}}^{-1}$ at the logarithmic mean time of $43 \mathrm{yr}$ after maximum) or

$\frac{\mathrm{d} M_{V}}{\mathrm{~d} \log t}=1.0 \pm 0.3$

which is entirely consistent with our prediction.

For completeness, we note that of course the white dwarf and the irradiated secondary star also will contribute to the observed visual light in post novae. We have used the code BINARY ++ written by A. van Teeseling (van Teeseling et al. 1998) to estimate these contributions for the parameters of V446 Her (Sect. 2). We find that the irradiated secondary is $\sim 2$ mag fainter than the irradiated accretion disc throughout the post-nova evolution. We estimate that today the variation of the geometric aspect of the irradiated secondary over the binary orbit produces a modulation of the visual brightness with a peak-to-peak amplitude of $\sim 0.3 \mathrm{mag}$. At optical wavelenghts, the hot white dwarf is more than 3 mag fainter than the disc, but is expected to be the dominant source of far-ultraviolet light in V446 Her. 


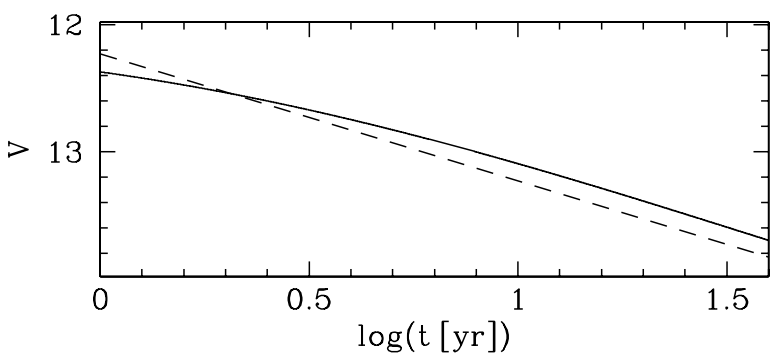

Fig. 5. The visual decline in post novae resulting from disc irradiation in a logarithmic scale. The solid line results from our detailed calculations (see Fig. 5b) and the dashed line represents our analytic expression given in Eq. (26).

\section{Discussion and conclusions}

In the previous section we calculated the visual decline in post nova systems resulting from decreasing disc irradiation and find it in agreement with the observed decline. This result has important implications for the post nova evolution.

One of the prominent working hypotheses, the hibernation scenario (see Shara 1989, for a review), interprets the observed visual fading in post novae as a result of decreasing mass transfer (caused by decreasing irradiation of the secondary). The hibernation scenario was evoked to solve discrepancies between the observations and the theory of the nova event, and to account for the low space density of CVs when compared to the number of nova eruptions.

The idea of irradiation-induced mass transfer enhancment is supported (a) by observations of the bright spot in the dwarf nova systems U Gem and Z Cha which indicate that the mass transfer rate is increased during an outburst (Smak 1995) and (b) by Kovetz et al. (1988) who calculated the effect of irradiation of the secondary star by the cooling white dwarf in post nova and find $\mathrm{d} \log \dot{M}_{\text {acc }} / \mathrm{d} \log t=-0.45$. Duerbeck (1992) used this result and d $M_{V} / \mathrm{d} \log \dot{M}_{\text {acc }} \simeq-1.56$ to obtain a visual decline rate of

$\mathrm{d} M_{V} / \mathrm{d} \log t=0.7$,

which was consistent with his data, Eq. (25), and which was thought to be a strong support for the hibernation scenario.

As cautious remarks we note (a) that the increased luminosity of the bright spot observed in U Gem and Z Cha may also be related to a decreased amount of stream overflow as the outer disc is geometrically thicker during an outburst (Smak 1985; Schreiber \& Hessman 1998; Hessman 1999) than in quiescence, and (b) that the irradiation induced mass transfer model of Kovetz et al. (1988) does not take into account shielding of the $L_{1}$ point by the accretion disc. Leibowitz et al. (1992) showed that a disc may be present in post novae already a few days after the explosion, and, hence, it seems plausible that the $L_{1}$ point is efficiently shielded in most, if not all nonmagnetic post novae. The only way to increase the mass transfer rate by irradiation on a short timescale even in the case of disc shielding is to assume meridional circulations which transport energy from the illuminated regions to the $L_{1}$-region (Sarna 1990; Kirbiyik \& Smith 1976; Kippenhahn \& Thomas 1979; Meyer \& Meyer-Hofmeister 1983). The process of meridional circulations is not well understood and, hence, the actual amount by which the mass transfer might be increased due to irradiation is very uncertain.

Our simulations of irradiated accretion discs have shown that the decrease of disc irradiation by the cooling white dwarf in post novae results in a decrease of the visual flux of post novae. Even though a decrease of the mass transfer rate may contribute to the observed long term visual decline in post novae, the effect of decreasing disc irradiation alone is sufficient to explain the observed decline rate - unless the reprocessing efficiency is extremely low (for $\beta=0.93, \log \left(\dot{M}\left[\mathrm{~g} \mathrm{~s}^{-1}\right]\right)=17.5, T_{\mathrm{wd}}(t=0)=$ $3.5 \times 10^{5} \mathrm{~K}$, and the parameter of V446 Her the decrease of the visual brightness is still 0.24 mag during the first 20 years after the hydrogen burning turned off).

Progress in our understanding of irradiated accretion discs requires an observational calibration of the reprocessing efficiency $(1-\beta)$, a venture that may be realized through a careful search for echo outbursts in the light curves of CVs containig hot white dwarfs, such as the post nova V446 Her, accompanied with the measurement of the primary's temperature.

Acknowledgements. Special thanks are extended to J. K. Cannizzo for providing us with a copy of his vertical structure code and to Brian Warner for helpful comments. MRS would like to thank the Deutsche Forschungsgemeinschaft (Ma 1545/2-1) and the DAAD (PKZ:D/01/05718) for financial support. BTG thanks for support from the DLR under grant 50 OR 99036.

\section{References}

Adams, F. C., Shu, F. H., \& Lada, C. J. 1988, ApJ, 326, 865

Cannizzo, J. K. 1993a, ApJ, 419, 318

Cannizzo, J. K. 1993b, in Accretion disks in compact stellar objects, ed. J. Wheeler, Advanced Ser. in Astrophys. and Cosmology No. 9 (Singapore: World Scientific), 6

Cannizzo, J. K., \& Cameron, A. G. W. 1988, ApJ, 330, 327

Cannizzo, J. K., \& Wheeler, J. C. 1984, ApJ, 55, 367

Dubus, G., Lasota, J., Hameury, J., \& Charles, P. 1999, MNRAS, 303, 139

Duerbeck, H. W. 1992, MNRAS, 258, 629

Gänsicke, B. T. 2000, Rev. Mod. Astron., 13, 151

Hameury, J., Menou, K., Dubus, G., Lasota, J., \& Hure, J. 1998, MNRAS, 298, 1048

Hameury, J. M., Lasota, J. P., \& Dubus, G. 1999, MNRAS, 303,39

Hameury, J. M., Lasota, J. P., \& Warner, B. 2000, A\&A, 353, 244

Hessman, F. V. 1999, ApJ, 510, 867

Honeycutt, R. K., Robertson, J. W., \& Turner, G. W. 1998, AJ, 115, 2527

King, A. R. 1997, MNRAS, 288, L16 
Kippenhahn, R., \& Thomas, H. 1979, A\&A, 75, 281

Kirbiyik, H., \& Smith, R. C. 1976, MNRAS, 176, 103

Kovetz, A., Prialnik, D., \& Shara, M. M. 1988, ApJ, 325, 828

Leibowitz, E. M., Mendelson, H., Mashal, E., Prialnik, D., \& Seitter, W. C. 1992, ApJ, 385, L49

Ludwig, K., \& Meyer, F. 1998, A\&A, 329, 559

Ludwig, K., Meyer-Hofmeister, E., \& Ritter, H. 1994, A\&A, 290, 473

Meyer, F., \& Meyer-Hofmeister, E. 1983, A\&A, 121, 29

Mineshige, S., Tuchman, Y., \& Wheeler, J. C. 1990, ApJ, 359, 176

Prialnik, D. 1986, ApJ, 310, 222

Sarna, M. J. 1990, A\&A, 239, 163

Schreiber, M. R., Gänsicke, B. T., \& Cannizzo, J. 2000a, A\&A, 362,268

Schreiber, M. R., Gänsicke, B. T., \& Hessman, F. V. 2000b, A\&A, 358, 221

Schreiber, M. R., \& Hessman, F. V. 1998, MNRAS, 301, 626
Shara, M. M. 1989, PASP, 101, 5

Shara, M. M., Livio, M., Moffat, A. F. J., \& Orio, M. 1986, ApJ, 311, 163

Sion, E. M. 1999, PASP, 111, 532

Smak, J. 1985, Acta Astron., 35, 351

Smak, J. 1989, Acta Astron., 39, 317

Smak, J. 1995, Acta Astron., 45, 355

Somers, M. W., \& Naylor, T. 1999, A\&A, 352, 563

Stienon, F. M. 1971, PASP, 83, 363

Suleimanov, V., Meyer, F., \& Meyer-Hofmeister, E. 1999, A\&A, 350, 63

Thorstensen, J. R., \& Taylor, C. 2000, MNRAS, 312, 629

Tuchman, Y., Mineshige, S., \& Wheeler, J. C. 1990, ApJ, 359, 164

van Teeseling, A., Reinsch, K., Pakull, M. W., \& Beuermann, K. 1998, A\&A, 338, 947

Warner, B. 1995, Cataclysmic Variable Stars (Cambridge: Cambridge University Press) 